\title{
Evaluación del deterioro del pavimento asfáltico en el tramo Ramal de Aspuzana-Nuevo Progreso mediante la metodología VIZIR en el ańo 2021
}

Evaluation of the asphalt pavement deterioration on the Aspuzana-
Nuevo Progreso branch using the VIZIR methodology in the year 2021

Recibido: octubre 22 de 2021 | Revisado: noviembre 10 de 2021 | Aceptado: noviembre 30 de 2021

\section{Gianmarco Herrera Suarez ${ }^{\mathrm{I}}$ Leonel Chahuares Paucar ${ }^{\mathrm{I}}$}

\begin{abstract}
Resumen
La evaluación de pavimentos permite entender el comportamiento de los deterioros superficiales a través del tiempo y optimizar costos en trabajos correctivos. El objetivo de esta investigación fue determinar el estado de deterioro del pavimento asfáltico aplicando la metodología VIZIR y plantear propuestas de rehabilitación. La técnica de recolección de datos consistió en la observación y como instrumentos de la metodología VIZIR se utilizó formatos de registro adoptados de la guía metodológica para el diseńo de obras de rehabilitación de pavimentos asfálticos de carreteras (INVIAS-2008). Centramos nuestra atención en la ruta nacional $5 \mathrm{~N}$ entre las localidades de Ramal de Aspuzana y Nuevo Progreso ubicadas en el distrito de Nuevo Progreso, provincia de Tocache, departamento de San Martín, con ancho promedio de 8.70 metros y longitud de 48.8 kilómetros. Los resultados obtenidos muestran que el estado de deterioro del pavimento evaluado es calificado como marginal según rangos del método VIZIR. A través de este método se determinó que en las unidades de muestra evaluadas existen dos deterioros de tipo A relacionados a la condición estructural con mayor grado de incidencia: Fisuras piel de cocodrilo $(35.2 \%)$, bacheos o parcheos $(35.6 \%)$ y la existencia de dos deterioros de tipo $\mathrm{B}$ relacionados a la condición funcional con mayor grado de incidencia: Pérdida de la película de ligante (26.2\%), pulimento de agregados (37.2\%). Se concluye que el tramo evaluado requiere de rehabilitación por refuerzo y reconstrucción debido a la calificación del pavimento como marginal además de estar expuesto a cargas y riesgo por drenaje superficial.
\end{abstract}

Palabras clave: Método VIZIR; deterioros; evaluación
Autor para correspondencia E-mail: gianmarcohs@upeu.edu.pe

\begin{abstract}
Pavement evaluation allows understanding the behavior of surface deterioration over time and optimizing costs in corrective work. The objective of this study was to determine the state of deterioration of the asphalt pavement applying the VIZIR methodology and to propose rehabilitation proposals. The data collection technique consisted of observation and as instruments of the VIZIR methodology registration formats adopted from the Methodological Guide for the design of rehabilitation works of asphalt road pavements (INVIAS-2008) were used. The study population was the national route $5 \mathrm{~N}$ between the towns of Ramal de Aspuzana and Nuevo Progreso located in the District of Nuevo Progreso Province of Tocache Department of San Martin with an average width of 8.70 meters and length of 48.8 kilometers. The results obtained show that the state of deterioration of the evaluated pavement is classified as marginal according to the ranges of the VIZIR method.
\end{abstract}

(C) Los autores. Este artículo es publicado por la Revista Campus de la Facultad de Ingeniería y Arquitectura de la Universidad de San Martín de Porres. Este artículo se distribuye en los términos de la Licencia Creative Commons Atribución No-comercial - Compartir-Igual 4.0 Internacional (https://creativecommons.org/licenses/ CC-BY), que permite el uso no comercial, distribución y reproducción en cualquier medio siempre que la obra original sea debidamente citada. Para uso comercial contactar a: revistacampus@usmp.pe.

https: 
Through this method it was determined that in the evaluated sample units there are 2 type A deteriorations related to the structural condition with the highest incidence crocodile skin fissures $(35.2 \%)$ potholes or patches $(35.6 \%)$ and the existence of 2 type $\mathrm{B}$ deteriorations related to the functional condition with a higher incidence Loss of the binder film (26.2\%) Aggregate polish (37.2\%). It was concluded that the evaluated section requires rehabilitation by reinforcement and reconstruction due to the classification of the pavement as marginal in addition to being exposed to loads and risk due to surface drainage.

Key words: VIZIR Method; deterioration; evaluation

\section{Introducción}

El buen servicio de un pavimento asfáltico se determina según la capacidad para proporcionar a los usuarios confort, garantía y economía. El nivel del servicio de los pavimentos asfálticos es afectado por los diferentes tipos de deterioros y según el nivel de gravedad. La recuperación del nivel de servicio de los pavimentos asfálticos requiere de una evaluación que incluye arte $\mathrm{y}$ ciencia debido a la inexistencia de ábacos comprobados o fórmulas como se tiene para el diseño. Por lo tanto, requiere de criterio del ingeniero para determinar la técnica más acertada a ejecutar en el proceso de rehabilitación del pavimento asfáltico considerando el tipo de deterioro que presenta el pavimento a intervenir (INVIAS, 2008).

La conservación de pavimentos es un conjunto de actividades de ingeniería vial, con la finalidad de evitar el deterioro prematuro de los elementos que conforman una vía asfáltica. Además, se debe considerar los aspectos económicos ejecutando las actividades de rehabilitación de manera eficiente y oportuna para minimizar gastos $y$ cumplir las metas que se programen según el periodo anual (MTC, 2018).
La aplicación del método VIZIR en la evaluación de deterioros de pavimentos asfálticos dispone una diferencia clara entre las fallas estructurales y funcionales, categorizando los deterioros en tipos A y $B$ que permite clasificar el estado de un pavimento mediante inspección visual (Rojas, 2011). Los deterioros de tipo A son las que caracterizan la condición estructural del pavimento, estos daños pueden ser ahuellamientos, deformaciones, agrietamientos relacionados con la fatiga del pavimento y con respectos a los deterioros de tipo B son los que caracterizan los daños funcionales, que pueden tener su origen en deficiencias constructivas o condiciones locales que se evidencias en la calzada.

La evaluación de pavimentos es necesaria e importante porque permite conocer los deterioros superficiales a través de tiempo para poder ejecutar correcciones adecuadas considerando el tiempo óptimo que permitirá la optimización de los costos por obras de rehabilitación, mejorando el nivel de servicio vial y ahorrando gastos mayores para los usuarios y la administración vial (Zevallos, 2018).

En el presente artículo, no solo se identifican los tipos de deterioros 
encontrados en el tramo de estudio. Además se proponen técnicas de rehabilitación, condición de drenaje superficial, causas que dieron origen a los deterioros y finalmente se presenta un mapa gráfico según categorización de deterioro.

\section{Métodos}

La presente investigación se desarrolló en la carretera nacional $5 \mathrm{~N}$ entre el tramo Ramal de Aspuzana y Nuevo Progreso, ubicada en el distrito de Nuevo Progreso, provincia de Tocache, departamento de San Martín (Figura 1). Los trabajos de inspección o campo que requiere el estudio se efectuaron en los meses de abril y mayo de 2021 .

El clima en la zona de estudio es característico de la selva: templado, con abundante humedad durante todas las estaciones del año con temperatura máxima de $35^{\circ} \mathrm{C}$, promedio de $25^{\circ} \mathrm{C}$ y mínima de $15^{\circ} \mathrm{C}$. La precipitación anual acumulada máxima es $5087.4 \mathrm{~mm}$, el promedio $4489.25 \mathrm{~mm}$ y el mínimo es $3952.2 \mathrm{~mm}$ (SENAMHI, 2021).

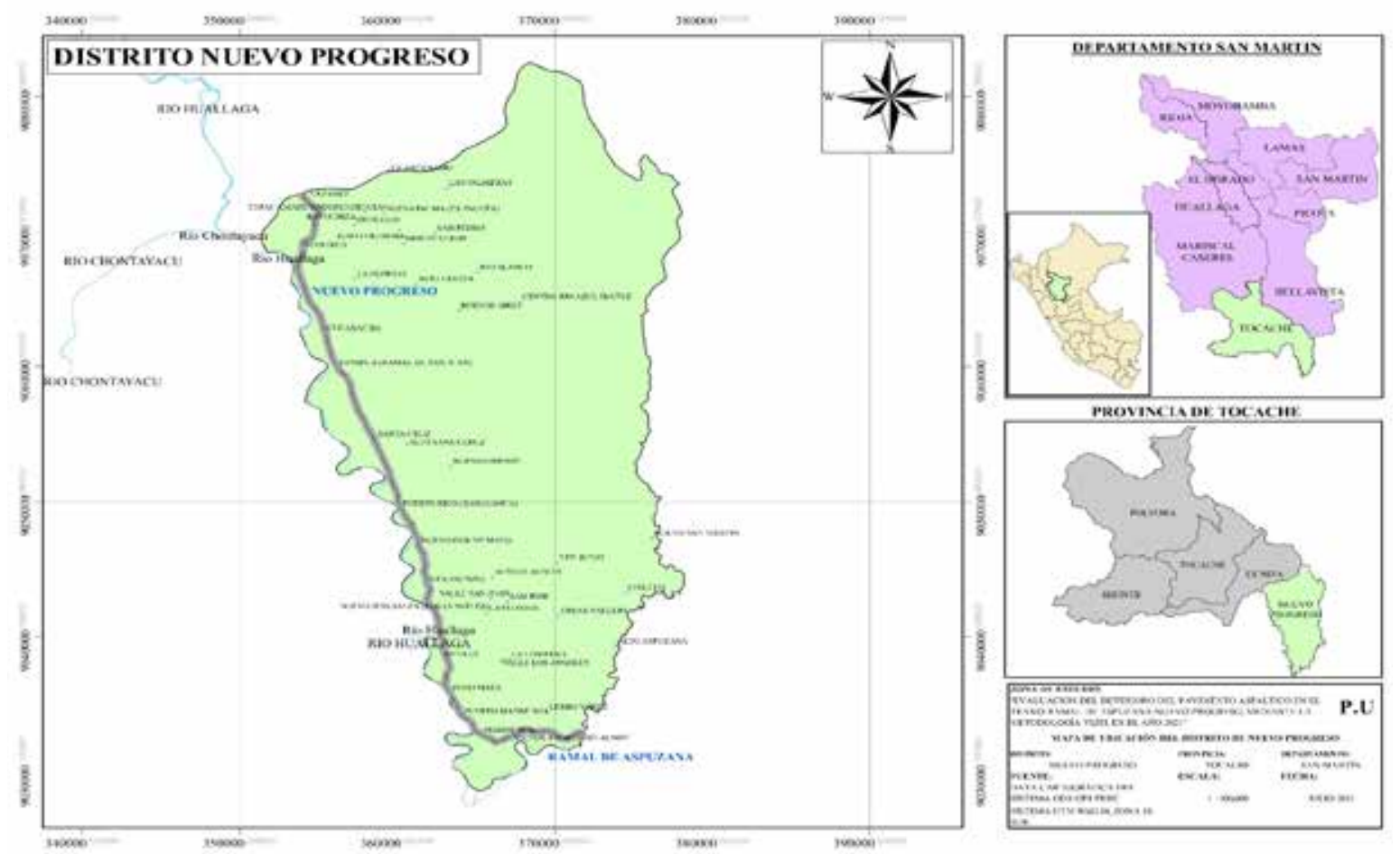

Figura 1. Ubicación del tramo en estudio

La investigación se desarrolló en tres pasos de acuerdo al esquema general que se presenta en la Figura 2. Cada paso identifica los componentes que se desarrollaron para la investigación, como en el paso 1 de los estudios de campo, el paso 2 de proceso de datos y finalmente, el paso 3 de modelación gráfica de los resultados de la evaluación. 


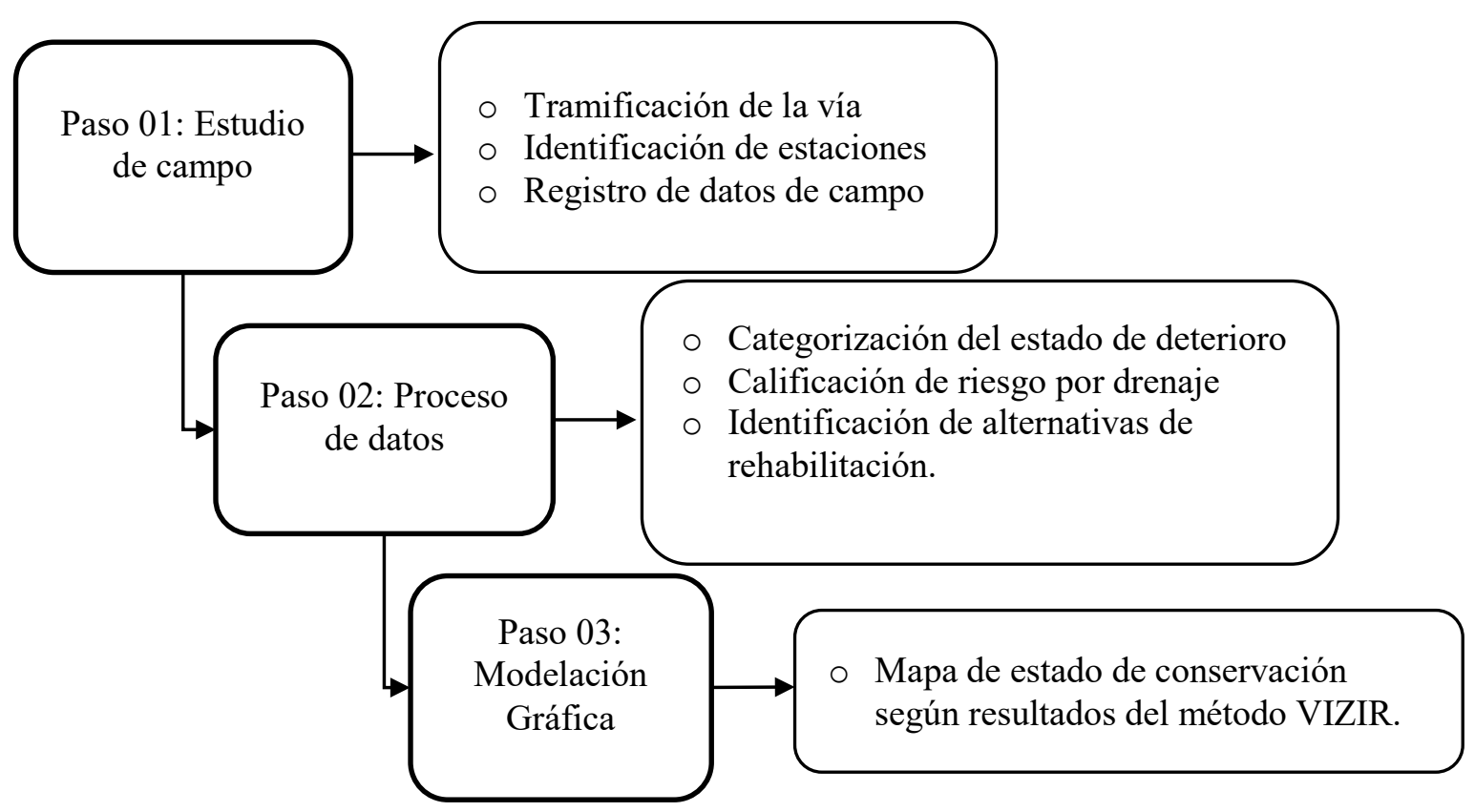

Figura 2. Esquema general de la metodología para el desarrollo de la investigación

\section{Estudio de campo}

La tramificación se refiere a la subdivisión del tramo a evaluar de acuerdo a los parámetros que permanecen en el tiempo, como estructura, tránsito y clima. Además, es necesario indicar que la metodología CONREVIAL es la más manejada en el Perú; sin embargo, no es una metodología patrón. La metodología de exploración es normalmente seleccionada por la empresa responsable de la auscultación de degradaciones generalmente según la experiencia y criterio del personal encargado (Hidalgo, 2006).

Considerando que el tramo en estudio corresponde a un solo tipo de estructura diseñada para tránsito ligero y pesado, además de considerar que en el tramo a evaluar predomina un solo clima denominado A(r) A' H4: (Muy húmedo lluvioso con precipitación abundante todo en año), se procedió con el primer paso de la investigación que consistió en la tramificación del tramo de estudio en 10 secciones de 1000 metros cada.

La longitud total del tramo es de 48,8 kilómetros, las distancias de las secciones se midieron en campo (Tabla 1) y de igual proceder se tomó las estaciones para el aforo vehicular (Tabla 2). Las coordenadas son parte del trabajo de campo, se obtuvo con GPS navegador y un cuentakilómetros de moto. 
Evaluación del deterioro de pavimento asfáltico en el tramo Ramal de Aspuzana - Nuevo Progreso MEDIANTE LA METODOLOGÍA VIZIR EN EL AÑO 202 I

Tabla 1

Tramificación de la vía a inspeccionar según el método VIZIR y riesgo por drenaje superficial

\begin{tabular}{|c|c|c|c|c|}
\hline & \multicolumn{3}{|c|}{ COORDENADAS } & \multirow{2}{*}{$\frac{\text { PROGRESIVA }}{\mathrm{km}}$} \\
\hline Secciones & Punto & Este $(\mathrm{m})$ & Norte $(\mathrm{m})$ & \\
\hline \multirow[t]{2}{*}{ Tramo I } & P1 & 370597 & 9032445 & $00+000$ \\
\hline & P2 & 369758 & 9032957 & $01+000$ \\
\hline \multirow[t]{2}{*}{ Tramo II } & P3 & 366159 & 9032241 & $05+000$ \\
\hline & P4 & 365339 & 9032744 & $06+000$ \\
\hline \multirow[t]{2}{*}{ Tramo III } & P5 & 363278 & 9036004 & $10+000$ \\
\hline & P6 & 363104 & 9036930 & $11+000$ \\
\hline \multirow[t]{2}{*}{ Tramo IV } & P7 & 362675 & 9040718 & $15+000$ \\
\hline & P8 & 362481 & 9041682 & $16+000$ \\
\hline \multirow[t]{2}{*}{ Tramo V } & P9 & 361807 & 9045297 & $20+000$ \\
\hline & P10 & 361620 & 9046252 & $21+000$ \\
\hline \multirow[t]{2}{*}{ Tramo VI } & P11 & 360056 & 9049842 & $25+000$ \\
\hline & P12 & 359926 & 9050818 & $26+000$ \\
\hline \multirow[t]{2}{*}{ Tramo VII } & P13 & 358519 & 9054606 & $30+000$ \\
\hline & P14 & 358074 & 9055502 & $31+000$ \\
\hline \multirow[t]{2}{*}{ Tramo VIII } & P15 & 356766 & 9059256 & $35+000$ \\
\hline & P16 & 356155 & 9060055 & $36+000$ \\
\hline \multirow[t]{2}{*}{ Tramo IX } & P17 & 355038 & 9063883 & $40+000$ \\
\hline & P18 & 354493 & 9064719 & $41+000$ \\
\hline \multirow[t]{2}{*}{ Tramo X } & P19 & 353997 & 9068520 & $45+000$ \\
\hline & P20 & 354299 & 9069400 & $46+000$ \\
\hline
\end{tabular}

Tabla 2

Selección de estaciones para aforo vehicular

\begin{tabular}{cccccc}
\hline No & ESTACIÓN & \multicolumn{2}{c}{ COORDENADAS } & PROGRESIVA & $\begin{array}{c}\text { CÓDIGO DE } \\
\text { ESTACIÓN }\end{array}$ \\
\hline & & Este $(\mathrm{m})$ & Norte $(\mathrm{m})$ & $\mathrm{km}$ & \\
1 & Ramal de Aspuzana & 370400 & 9032480 & $0+100$ & E-001 \\
\hline 2 & Madre Mía & 365839 & 9032549 & $5+500$ & E-002 \\
3 & Sitully & 363332 & 9037865 & $12+000$ & E-003 \\
\hline 4 & San Jacinto & 362011 & 9043281 & $18+000$ & E-004 \\
5 & Santa Cruz & 358519 & 9054606 & $30+000$ & E-005 \\
\hline 6 & Nuevo Progreso & 355038 & 9063883 & $40+000$ & E-006 \\
7 & La Florida & 354112 & 9069301 & $46+000$ & E-007 \\
\hline
\end{tabular}

Como instrumento para aplicar la metodología VIZIR, se utiliza un formato de registro de deterioros de tipo $\mathrm{A}$ y $\mathrm{B}$, el cual incluye la calificación del nivel de gravedad, la longitud, área y en los casos que lo requiera la profundidad. La recopilación de información de campo se obtuvo entre los meses de abril y mayo 
de 2021. Se menciona la fecha porque es necesario tener en conocimiento como es el comportamiento de los deterioros a través del tiempo.

\section{Determinación del estado superficial según el Método VIZIR (Visión Inspección de Zonas e Itinerarios en Riesgo)}

La metodología (VIZIR) se originó en Francia a partir de los ańos 60 para pavimentos flexibles "Visión Inspection de Zones et Itinéraires Á Risque", y fue publicada por el laboratorio central de puentes y carreteras "Laboratoire Central des Ponts et Chaussées (LCPC)" (VIZIR, 1996).

El método VIZIR es considerado una herramienta para realzar una evaluación visual de las degradaciones en los pavimentos flexibles y estos pueden clasificarse como dańos estructurales y daños superficiales, además de contemplar la gravedad y extensión de las degradaciones (Unidad de Gestión Municipal, 2012).

Los deterioros son alteraciones producidas en la superficie de un pavimento asfáltico que pueden ser detectados visualmente y pueden producirse por acciones de las cargas de tránsito, por las condiciones climáticas, deficiencias en el proceso de producción y construcción, así también puede ser por la calidad de los materiales (Leguízamo, 2008). Los deterioros de acuerdo a la metodología VIZIR se clasifican y cuantifican en Tipo "A", que caracteriza los deterioros que afectan la condición estructural y en Tipo" B", los que caracterizan los deterioros que afecta la condición funcional.

Los tipos de deterioros juntamente con su nivel de gravedad $(1,2$ o 3) y descripción se observan en la Tabla 2, para deterioros de Tipo "A" y en la Tabla 3 para deterioros de Tipo "B".

Tabla 3

Descripción y niveles de gravedad de deterioros Tipo $A$

\begin{tabular}{|c|c|c|c|}
\hline & \multicolumn{3}{|c|}{ NIVEL DE GRAVEDAD } \\
\hline DETERIORO & 1 & 2 & 3 \\
\hline $\begin{array}{l}\text { Ahuellamiento y otras } \\
\text { deformaciones } \\
\text { estructurales }\end{array}$ & $\begin{array}{l}\text { Sensible al usuario, } \\
\text { pero poco } \\
\text { importante } \\
\text { Prof. }<20 \mathrm{~mm}\end{array}$ & $\begin{array}{l}\text { Deformaciones importantes. } \\
\text { Hundimientos localizados o } \\
\text { ahuellamientos. } \\
20 \mathrm{~mm} \leq \text { Prof. } \leq 40 \leq \mathrm{mm}\end{array}$ & $\begin{array}{l}\text { Deformaciones que afectan } \\
\text { de manera importante la } \\
\text { comodidad y la seguridad de } \\
\text { los usuarios. } \\
\text { Prof. > } 40 \mathrm{~mm}\end{array}$ \\
\hline $\begin{array}{l}\text { Fisuras } \\
\text { longitudinales } \\
\text { por fatiga }\end{array}$ & $\begin{array}{l}\text { Fisuras finas en la } \\
\text { huella de } \\
\text { rodamiento. } \\
<6 \mathrm{~mm}\end{array}$ & $\begin{array}{l}\text { Fisuras abiertas y a } \\
\text { menudo ramificadas. }\end{array}$ & $\begin{array}{l}\text { Fisuras muy ramificadas, } \\
\text { y/o muy abiertas. } \\
\text { Bordes de fisuras } \\
\text { ocasionalmente degradados. }\end{array}$ \\
\hline Piel de cocodrilo & $\begin{array}{l}\text { Piel de cocodrilo } \\
\text { formada por mallas } \\
\text { (> } 500 \mathrm{~mm} \text { ) con } \\
\text { fisuración fina, sin } \\
\text { pérdida de materiales. }\end{array}$ & $\begin{array}{l}\text { Mallas más densas } \\
(<500 \mathrm{~mm}) \text {, con pérdidas } \\
\text { ocasionales de materiales, } \\
\text { desprendimientos y ojos de } \\
\text { pescado en formación. }\end{array}$ & $\begin{array}{l}\text { Mallas con grietas muy } \\
\text { abiertas y con fragmentos } \\
\text { separados. } \\
\text { Las mallas son muy densas } \\
(<200 \mathrm{~mm}) \text {, con pérdida } \\
\text { ocasional ogeneralizada de } \\
\text { materiales. }\end{array}$ \\
\hline \multirow[t]{2}{*}{ Bacheos y Parcheos } & \multirow{2}{*}{$\begin{array}{l}\text { Intervención de } \\
\text { superficie ligada a } \\
\text { deterioros tipo B. }\end{array}$} & \multicolumn{2}{|c|}{ Intervenciones ligadas a deterioros tipo A } \\
\hline & & $\begin{array}{l}\text { Comportamiento } \\
\text { satisfactorio de la reparación. }\end{array}$ & $\begin{array}{l}\text { Ocurrencia de fallas en las } \\
\text { zonas reparadas. }\end{array}$ \\
\hline
\end{tabular}


Tabla 4

Descripción y niveles de gravedad de deterioros Tipo B

\begin{tabular}{|c|c|c|c|c|}
\hline \multirow{2}{*}{\multicolumn{2}{|c|}{ DETERIORO }} & \multicolumn{3}{|c|}{ NIVEL DE GRAVEDAD } \\
\hline & & 1 & 2 & 3 \\
\hline \multicolumn{2}{|c|}{$\begin{array}{l}\text { Fisura longitudinal de junta } \\
\text { deconstrucción }\end{array}$} & $\begin{array}{l}\text { Fina y única }<6 \\
\mathrm{~mm}\end{array}$ & $\begin{array}{l}\text {-Ancha }(\leq 6 \mathrm{~mm}) \text { sin } \\
\text { desprendimiento o } \\
\text { - Fina ramificada }\end{array}$ & $\begin{array}{l}\text { Ancha }(\leq 6 \mathrm{~mm}) \text { con } \\
\text { desprendimientos o } \\
\text { ramificada }\end{array}$ \\
\hline \multicolumn{2}{|c|}{ Fisuras de contracción térmica } & $\begin{array}{l}\text { Fisuras finas } \\
<6 \mathrm{~mm}\end{array}$ & $\begin{array}{l}\text { Anchas }(\leq 6 \mathrm{~mm}) \text { sin } \\
\text { desprendimiento, o } \\
\text { finas con } \\
\text { desprendimientos o } \\
\text { fisuras ramificadas }\end{array}$ & $\begin{array}{l}\text { Anchas }(\leq 6 \mathrm{~mm}) \text { con } \\
\text { desprendimientos }\end{array}$ \\
\hline \multicolumn{2}{|l|}{ Fisuras parabólicas } & $\begin{array}{l}\text { Fisuras finas } \\
<6 \mathrm{~mm}\end{array}$ & $\begin{array}{l}\text { Anchas }(\leq 6 \mathrm{~mm}) \sin \\
\text { desprendimientos }\end{array}$ & $\begin{array}{l}\text { Anchas }(\leq 6 \mathrm{~mm}) \text { con } \\
\text { desprendimientos }\end{array}$ \\
\hline \multicolumn{2}{|l|}{ Fisuras de borde } & $\begin{array}{l}\text { Fisuras finas } \\
<6 \mathrm{~mm}\end{array}$ & $\begin{array}{l}\text { Anchas }(\leq 6 \mathrm{~mm}) \sin \\
\text { desprendimientos }\end{array}$ & $\begin{array}{l}\text { Anchas }(\leq 6 \mathrm{~mm}) \text { con } \\
\text { desprendimientos }\end{array}$ \\
\hline \multicolumn{2}{|l|}{ Abultamientos } & $\mathrm{h}<20 \mathrm{~mm}$ & $20 \mathrm{~mm} \leq \mathrm{h} \leq 40 \mathrm{~mm}$ & $\mathrm{~h}>40 \mathrm{~mm}$ \\
\hline \multirow{2}{*}{$\begin{array}{l}\text { Ojos de pescado* } \\
100 \mathrm{~m}\end{array}$} & Cantidad & $<5$ & 5 a $10<5$ & $>10 \quad 5$ a 10 \\
\hline & $\varnothing(\mathbf{m m})$ & $\leq 300$ & $\leq 1000$ & $\leq 300 \leq 1000$ \\
\hline \multicolumn{2}{|c|}{$\begin{array}{l}\text { Desprendimientos: } \\
\text { - Pérdida de película ligante } \\
\text { - Pérdida de agregado }\end{array}$} & Pérdidas aisladas & Pérdidas continuas & $\begin{array}{l}\text { Pérdidas generalizadas y } \\
\text { muy marcadas }\end{array}$ \\
\hline \multirow[t]{2}{*}{ Descascaramiento } & Prof. (mm) & $\leq 25$ & $\leq 25$ & $>25$ \\
\hline & Área (m2) & $\leq 0.8$ & $\leq 0.8$ & $>0.8$ \\
\hline \multicolumn{2}{|c|}{ Pulimentos agregados } & $\begin{array}{l}\text { Long. } \\
\text { Comprometida }< \\
10 \% \text { de la sección } \\
(100 \mathrm{~m}) .\end{array}$ & $\begin{array}{l}\text { Long. Comprometida } \\
\geq 10 \% \text { a }<50 \% \text { de la } \\
\text { sección }(100 \mathrm{~m})\end{array}$ & $\begin{array}{l}\text { Long. Comprometida } \\
>50 \% \text { de la sección }(100 \mathrm{~m})\end{array}$ \\
\hline \multicolumn{2}{|l|}{ Exudación } & $\begin{array}{l}\text { Puntual, área } \\
\text { específica }\end{array}$ & $\begin{array}{l}\text { Continúa sobre las } \\
\text { trayectorias por donde } \\
\text { circulan las ruedas del } \\
\text { vehículo. }\end{array}$ & $\begin{array}{l}\text { Continua y muy } \\
\text { marcada, en diversas } \\
\text { aéreas. }\end{array}$ \\
\hline \multicolumn{2}{|c|}{$\begin{array}{l}\text { Afloramientos: De mortero y de } \\
\text { agua }\end{array}$} & $\begin{array}{l}\text { Localizados } \\
\text { y apenas } \\
\text { perceptibles }\end{array}$ & Intensos & Muy intensos \\
\hline \multicolumn{2}{|c|}{$\begin{array}{l}\text { Desintegración de los bordes del } \\
\text { pavimento }\end{array}$} & $\begin{array}{l}\text { Inicio de la } \\
\text { desintegración, } \\
\text { sectores } \\
\text { localizados }\end{array}$ & $\begin{array}{l}\text { La calzada ha sido } \\
\text { afectada en un ancho } \\
\text { de } 500 \text { mm o más. }\end{array}$ & $\begin{array}{l}\text { Erosión extrema que } \\
\text { conduce a la } \\
\text { desintegración del } \\
\text { revestimiento asfáltico }\end{array}$ \\
\hline \multicolumn{2}{|c|}{$\begin{array}{l}\text { Escalonamiento entre calzadas y } \\
\text { berma }\end{array}$} & $\begin{array}{l}\text { Desnivel entre } 10 \\
\mathrm{~mm} \text { a } 50 \mathrm{~mm}\end{array}$ & $\begin{array}{l}\text { Desnivel entre } 50 \text { y } 100 \\
\mathrm{~mm}\end{array}$ & $\begin{array}{l}\text { Desnivel superior a } 100 \\
\mathrm{~mm}\end{array}$ \\
\hline \multicolumn{2}{|c|}{ Erosión de las bermas } & Erosión incipiente & Erosión pronunciada & $\begin{array}{l}\text { La erosión pone en } \\
\text { peligro la estabilidad de la } \\
\text { calzada y la seguridad } \\
\text { de los usuarios. }\end{array}$ \\
\hline \multicolumn{2}{|l|}{ Segregación } & $\begin{array}{l}\text { Longitud } \\
\text { comprometida < } \\
10 \% \text { de la sección } \\
(100 \mathrm{~m}) .\end{array}$ & $\begin{array}{l}\text { Long. comprometida } \\
\geq 10 \% \text { a }<50 \% \text { de la } \\
\text { sección }(100 \mathrm{~m})\end{array}$ & $\begin{array}{l}\text { Long. comprometida > } \\
50 \% \text { de la sección }(100 \mathrm{~m})\end{array}$ \\
\hline
\end{tabular}

${ }^{*}$ Cuando el número de ojo de pescado supere el número y tamaño descrito en la tabla, se deberá considerar como un deterioro del tipo A. 
El método VIZIR para calificar el deterioro superficial de un pavimento asfáltico hace uso de dos índices: el índice de fisuración (If) que está relacionado a los agrietamientos de tipo estructural, y el índice de deformación (Id), está relacionado a las deformaciones de tipo estructural, ambos índices permiten determinar el índice de deterioro superficial (Is) según la extensión y gravedad (Porta, 2016).

La rehabilitación de deterioros de tipo A aporta tácitamente el atenuante para los defectos de tipo B, de manera que los defectos de este tipo solo se intervienen en ausencia de defectos de tipo A. Por tal motivo en la calificación del Índice de deterioro superficial (Is) solo se considera los deterioros de tipo A. Inicialmente se determina el Índice de fisuración (If) y el Índice de deformación (Id), los cuales están valorados cuantitativamente en función a su gravedad y extensión de fisuras o deformaciones estructurales. El acoplamiento de ambos índices genera un primer índice de calificación, el cual es corregido en función de la extensión y calidad de los trabajos de parcheo y bacheo, una vez ejecutada la corrección se determina el Índice de deterioro superficial (Is), el cual califica la sección escogida para el cálculo (Instituto Nacional de Vias, 2008).

A partir del Índice de deterioro superficial (Is), se categoriza en tres la condición del pavimento. Valores de (Is) 1 y 2 representan pavimentos con muy pocos fisuramientos y deformaciones, los valores de (Is) 3 y 4 muestran pavimentos con fisuras de origen estructural y limitadas deformaciones y finalmente los valores de (Is) 5, 6 y 7 son pavimentos con abundancia de fisuras y deformaciones estructurales (Apolinario, 2012).

\section{Inspección y establecimientos de niveles de riego por drenaje superficial}

Las fisuras en los pavimentos facilitan la infiltración del agua que acelera la extensión y gravedad de los fenómenos de degradación en los pavimentos, llevando a la aparición de bordes agrietados, desprendimiento de material hasta la posterior formación de ojos de pescado (Echeverry, 2004).

Para determinar el nivel de riesgo por drenaje superficial se consideran tres parámetros básicos para valorar los dańos causados por el agua. La Impermeabilidad del revestimiento (R). Recopila información de la superficie del pavimento, para ver la impermeabilidad que tiene la calzada. $\mathrm{R}=0, \mathrm{R}=1, \mathrm{y} \mathrm{R}=2$, Drenaje superficial (A). Recopila información del diseño geométrico de la vía y de las estructuras de drenaje superficial. $A=0, A=1 \quad y$ $A=2$, Ambiente hidrogeológico del pavimento $(\mathrm{H})$. Registra los posibles efectos de degradación que puede tener las estructuras del pavimento debido a las corrientes de agua temporales y no temporales sobre la carpeta asfáltica o en los laterales del pavimento. $\mathrm{H}=0, \mathrm{H}=1 \mathrm{y}$ $\mathrm{H}=2$ (Gonzáles, 2008). La calificación final se muestra en la Tabla 5. 
Evaluación del deterioro de pavimento asfáltico en el tramo Ramal de Aspuzana - Nuevo Progreso MEDIANTE LA METODOLOGÍA VIZIR EN EL AÑO 202 I

Tabla 5

Calificación de niveles de riesgo por drenaje superficial

\begin{tabular}{cll}
\hline CALIFICACIÓN & \multicolumn{1}{c}{ RIESGO } \\
\hline $0-2$ & N (NO HAY RIESGO) & $\begin{array}{l}\text { Condiciones no propicias para que se presente daño en la } \\
\text { estructura por causa del agua. }\end{array}$ \\
$3-4$ & B (BAJO RIESGO) & $\begin{array}{l}\text { Condiciones que pueden generar daño en la estructura por } \\
\text { efectos del agua. }\end{array}$ \\
$5-6$ & $\begin{array}{l}\text { E (ELEVADO DE } \\
\text { RIESGO) }\end{array}$ & $\begin{array}{l}\text { Condiciones determinantes para causar la aparición y rápida } \\
\text { degradación de la estructura por presencia del agua. }\end{array}$ \\
\hline
\end{tabular}

\section{Selección de técnicas de rehabilitación}

Las técnicas más comunes de rehabilitación consisten en la colocación de sobrecapas y en muchas ocasiones no se ha considerado el estado y capacidad estructural del pavimento. Es necesario que la corrección de los deterioros existentes mejore el estado estructural, funcional $y$ drenaje del pavimento (Instituto Nacional de Vias, 2008). Las alternativas de rehabilitación de los pavimentos asfálticos están agrupadas en las $4 \mathrm{R}$ (restauración, refuerzo, reciclado y reconstrucción) de acuerdo a la Figura 3, y las posibles causas de deterioros según técnica de rehabilitación en la Figura 4.

\begin{tabular}{|c|c|c|c|c|c|c|c|c|c|c|c|c|c|c|c|c|c|c|c|c|c|c|c|c|c|c|c|}
\hline \multirow[b]{2}{*}{ 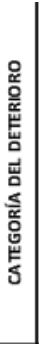 } & \multirow[b]{2}{*}{ TIPO DE DETERIORO } & \multicolumn{7}{|c|}{ R1 } & \multicolumn{2}{|c|}{$\begin{array}{c}\text { R2 } \\
\text { REFUERZO }\end{array}$} & \multicolumn{3}{|c|}{$\begin{array}{c}\text { R3 } \\
\text { RECICLADO }\end{array}$} & \multicolumn{2}{|c|}{\begin{tabular}{|c|} 
R4 \\
BECONSTRUCCIÓ
\end{tabular}} & \multicolumn{12}{|c|}{ TRATAMIENTOS PREVIOS } \\
\hline & & 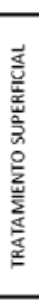 & 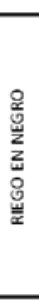 & 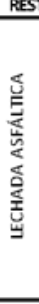 & 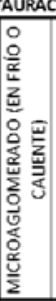 & 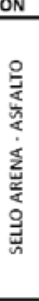 & 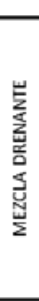 & 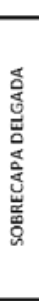 & 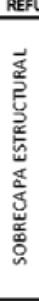 & 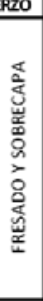 & 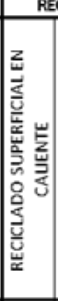 & 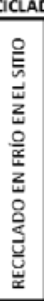 & 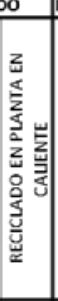 & 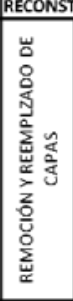 & 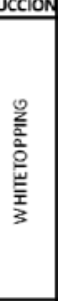 & 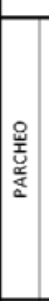 & 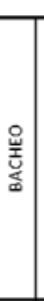 & 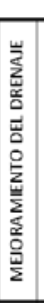 & 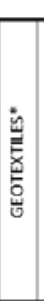 & 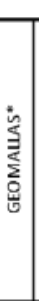 & $\sum_{\text {s }}$ & 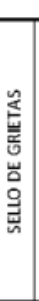 & 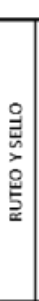 & 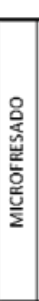 & 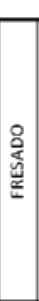 & 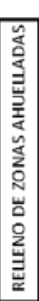 & 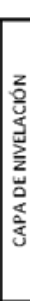 \\
\hline \multirow{6}{*}{$\frac{n}{\frac{n}{2}}$} & Longitudinal & $\mathrm{x}$ & & 0 & 0 & 0 & $x$ & & $\cdot$ & 0 & $x$ & $x$ & $x$ & $x$ & $\mathrm{x}$ & $x$ & $x$ & $x$ & • & - & $x$ & 0 & $x$ & & & & \\
\hline & Piel cocodrilo & & & & & & $x$ & & • & $\mathrm{x}$ & $x$ & • & - & - & - & & - & 0 & 0 & $\cdot$ & $x$ & 0 & & & 0 & & \\
\hline & Transversal & $\mathrm{x}$ & & 0 & 0 & & & & • & • & - & 0 & - & 0 & 0 & 0 & 0 & & - & - & • & • & • & 0 & 0 & & \\
\hline & Reflexión & $\mathrm{x}$ & & 0 & 0 & & & & $\cdot$ & - & - & 0 & - & 0 & 0 & 0 & 0 & & • & - & • & • & • & 0 & 0 & & \\
\hline & Bloque & $\mathrm{x}$ & & 0 & 0 & & & & 0 & - & - & 0 & - & 0 & 0 & & & & • & $\cdot$ & • & $\cdot$ & 0 & 0 & 0 & & \\
\hline & Borde & 0 & & 0 & 0 & & & & 0 & • & 0 & 0 & 0 & & & & • & 0 & 0 & 0 & & 0 & & & & & \\
\hline \multirow{5}{*}{ 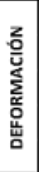 } & Ahuel la miento & & & & $x$ & & & & $\cdot$ & - & $\cdot$ & 0 & - & • & - & • & - & 0 & & & & & & 0 & • & • & • \\
\hline & Ondulaciones & & & & & & & - & 0 & - & - & 0 & 0 & 0 & 0 & - & 0 & & & & & & & • & • & & 0 \\
\hline & \begin{tabular}{|l} 
Levantamientos \\
\end{tabular} & & & & & & & 0 & $\cdot$ & $\cdot$ & 0 & $\cdot$ & $\cdot$ & $\cdot$ & $\cdot$ & $\cdot$ & • & & & & & & & & • & & $\cdot$ \\
\hline & Depresiones & & & & & & & 0 & 0 & 0 & & 0 & 0 & $\cdot$ & - & 0 & - & 0 & & & & & & & • & 0 & $\cdot$ \\
\hline & Resalto en sobrecapas & & & 0 & $\bullet$ & & & & 0 & & & 0 & 0 & 0 & 0 & 0 & • & & $\cdot$ & & 0 & & 0 & • & • & & \\
\hline \multirow{6}{*}{ 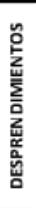 } & Descas caramiento & & & & & & & & $\cdot$ & & & 0 & $\cdot$ & 0 & 0 & & $\cdot$ & & & 0 & & & & & & & \\
\hline & Ojo de pescado & & & & & & & & $\cdot$ & 0 & & 0 & - & 0 & 0 & 0 & • & & 0 & $\cdot$ & & & & & & & \\
\hline & \begin{tabular}{|l} 
Pérdida de agr egados \\
\end{tabular} & $\cdot$ & 0 & - & $\bullet$ & 0 & $\cdot$ & $\cdot$ & 0 & $\cdot$ & • & 0 & - & 0 & 0 & - & • & 0 & • & $\cdot$ & & & & 0 & • & & \\
\hline & Pérdida de ligante & & & & & & & & $\cdot$ & & & • & - & 0 & 0 & & • & & 0 & 0 & & & & & & & \\
\hline & Pulimento de agrega dos & • & & - & - & 0 & • & 0 & 0 & - & - & & 0 & 0 & 0 & - & 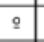 & & & & & & & • & • & & \\
\hline & Bombeo & & & & & & & & 0 & & & - & - & - & - & & • & & 0 & 0 & & & & & & & \\
\hline \multirow{3}{*}{ 莒 } & Segregación & & & & & & & 0 & 0 & 0 & 0 & • & • & 0 & 0 & 0 & • & & 0 & 0 & & & & & & & \\
\hline & Cuarteado & & & & & & & - & 0 & 0 & - & 0 & - & 0 & 0 & - & - & & & & & & & • & • & & \\
\hline & Exudación & & & 0 & 0 & & - & & 0 & 0 & 0 & 0 & - & 0 & 0 & 0 & • & & & 0 & & & & 0 & • & & \\
\hline \multicolumn{28}{|c|}{$\begin{array}{l}\text { - Iratamiento más adecuado } \\
\text { Se requieren técnicas o prod }\end{array}$} \\
\hline
\end{tabular}

Figura 3. Técnicas de rehabilitación y posibles causas 


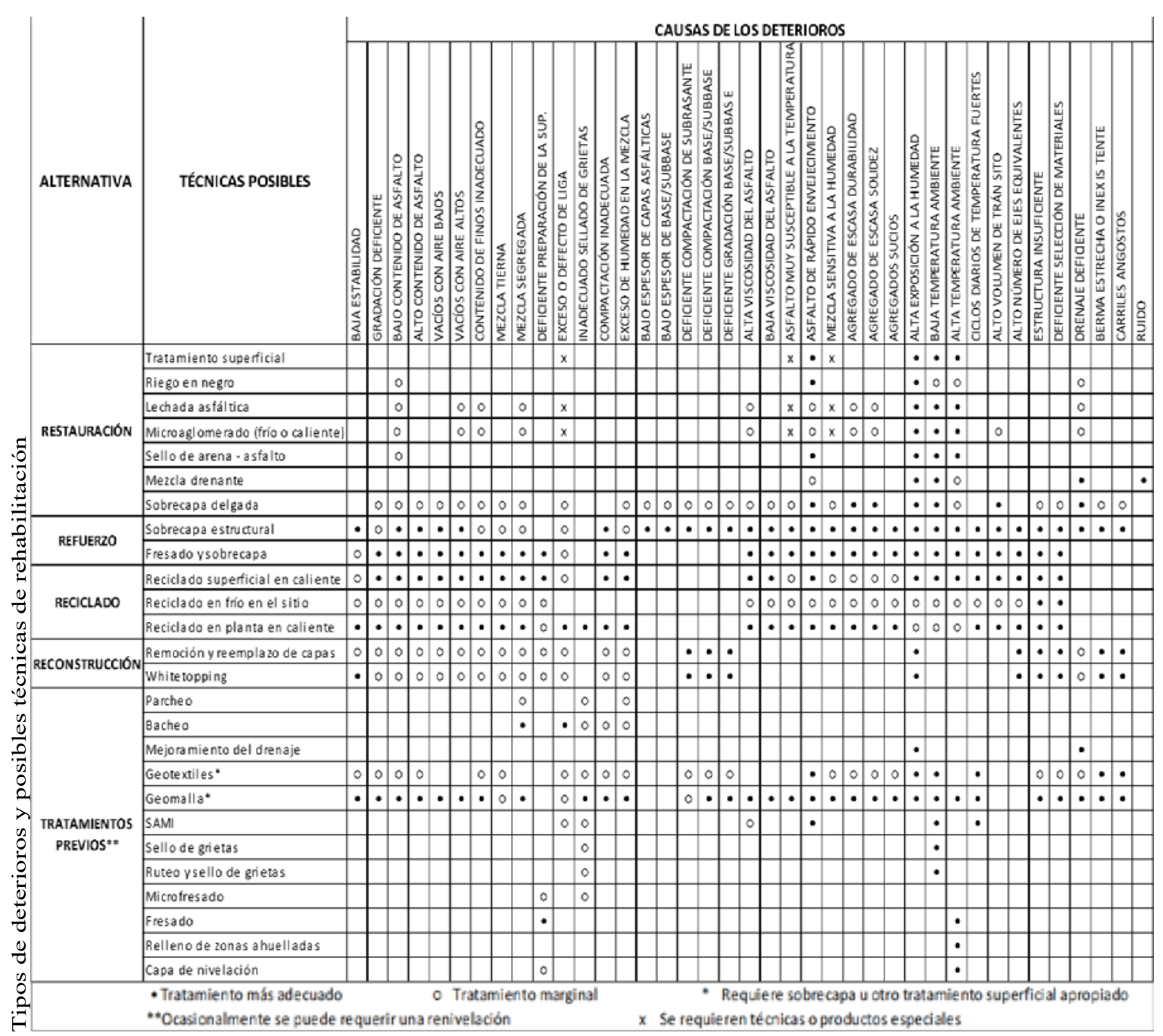

Figura 4. Tipos de deterioros y posibles técnicas de rehabilitación

Los pavimentos de las carreteras se someten a un proceso de deterioro no lineal desde el inicio de su vida útil, pero a diferencia de otros activos civiles, los pavimentos sobre el suelo se pueden tratar fácilmente para un determinado lugar y condición específica a través de mantenimiento, rehabilitación o tratamiento de reconstrucción (Kelly, Dalaney, Chai, \& Mohamed, 2014). Para definir los trabajos de rehabilitación de los pavimentos asfálticos también es necesario tener en cuenta la composición del tránsito mediante la determinación del volumen vehicular en el tramo a evaluar. La distribución vehicular se clasifica en vehículos ligeros que comprenden autos, station wagon, camionetas, micro y buses de dos ejes y mayor a tres ejes, vehículos pesados son considerados los camiones de dos, tres y cuatro ejes, los semi trayler y finalmente están los trayler.

\section{Mapa de estado de conservación según resultados del método VIZIR}

El mapa del estado de conservación del tramo evaluado es la representación de la calificación y estaciones de inspección de campo de acuerdo a las secciones calculadas con la metodología VIZIR, es presentado conforme a los resultados del Índice de deterioro superficial (Is) obtenidos en la evaluación, también se 
representa los resultados por nivel de riesgo por drenaje superficial.

La condición del pavimento en el tramo evaluado está representada según los tres niveles de Índice de deterioro superficial (Is) (buena, marginal o regular y deficiente), a la vez serán descritos con los colores verde, amarillo y rojo.

\section{Resultados y Discusión}

Los resultados obtenidos se presentan siguiendo la secuencia del esquema de metodología.

En primer lugar, se identifica los deteriorosexistentes en el tramo de estudio tanto para los de tipo A y los de tipo B con su respectivo dato de incidencia. En segundo lugar, se muestran los valores calculados según la metodología VIZIR en los diferentes tramos evaluados con sus respectivos datos de incidencia en el consolidado de categorización; en tercer lugar, se presentan los resultados del establecimiento de nivel de riesgos por drenaje en el tramo de estudio con su respectivo dato de incidencia. En el cuarto lugar, se presenta las técnicas de rehabilitación seleccionadas juntamente con las posibles causas de los deterioros y su comparación económica de estado de deterioro con la rehabilitación y finalmente se presenta el mapa del estado de deterioro del tramo evaluado en la investigación con su respectiva leyenda.

\section{Fallas existentes en el tramo de estudio}

En el recorrido del tramo evaluado se han encontrado deterioros de tipo A (degradación estructural) y de tipo $\mathrm{B}$ (degradación funcional), de los cuales las fallas de tipo A que presentan mayor incidencia son los deterioros de bacheo y parcheo (B) con $35.6 \%$ como se observa en la Figura 5 y las fallas de tipo B que presenta mayor incidencia es el deterioro de pulimentos de agregados (PU) como se observar en la Figura 6.

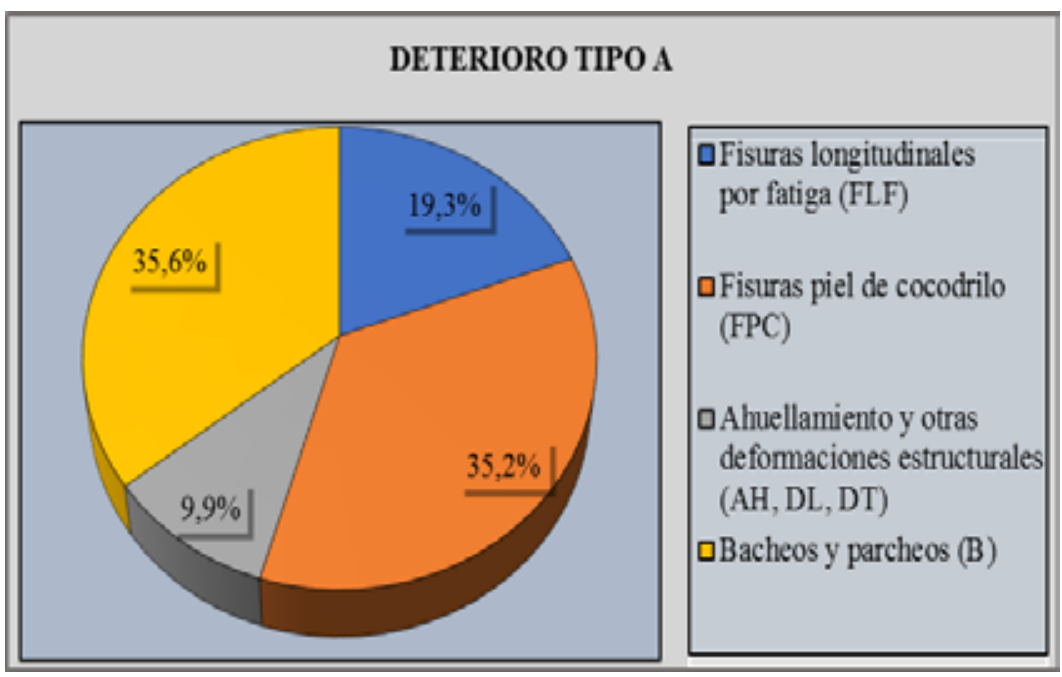

Figura 5. Deterioros de tipo A registrados en el tramo de estudio 


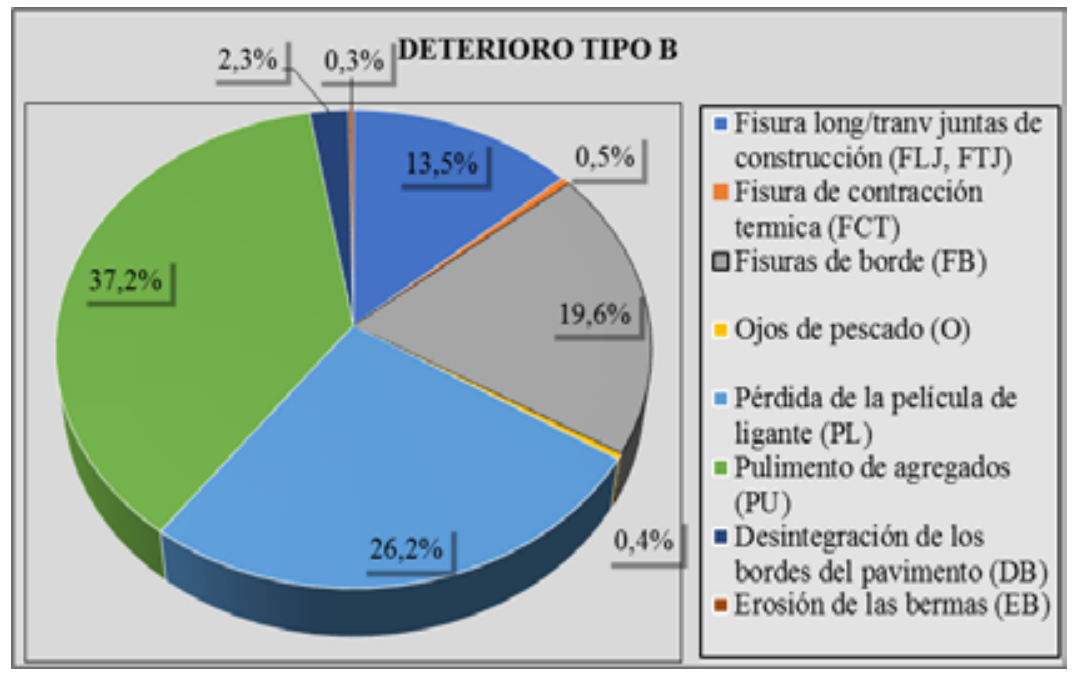

Figura 6. Deterioros de tipo B registrados en el tramo de estudio

Valores de Índice de deterioro superficial (Is) por tramo según el método VIZIR

El tramo evaluado presenta un estado de deterioro marginal o también considerado regular según los siguientes porcentajes obtenidos del Índice de deterioro superficial (Is): Categoría buena $25.0 \%$, categoría marginal $42.5 \%$ y categoría deficiente $32.5 \%$. El resultado del tramo evaluado se presenta en la Figura 7 según la categorización final del estado de deterioro según la metodología VIZIR.

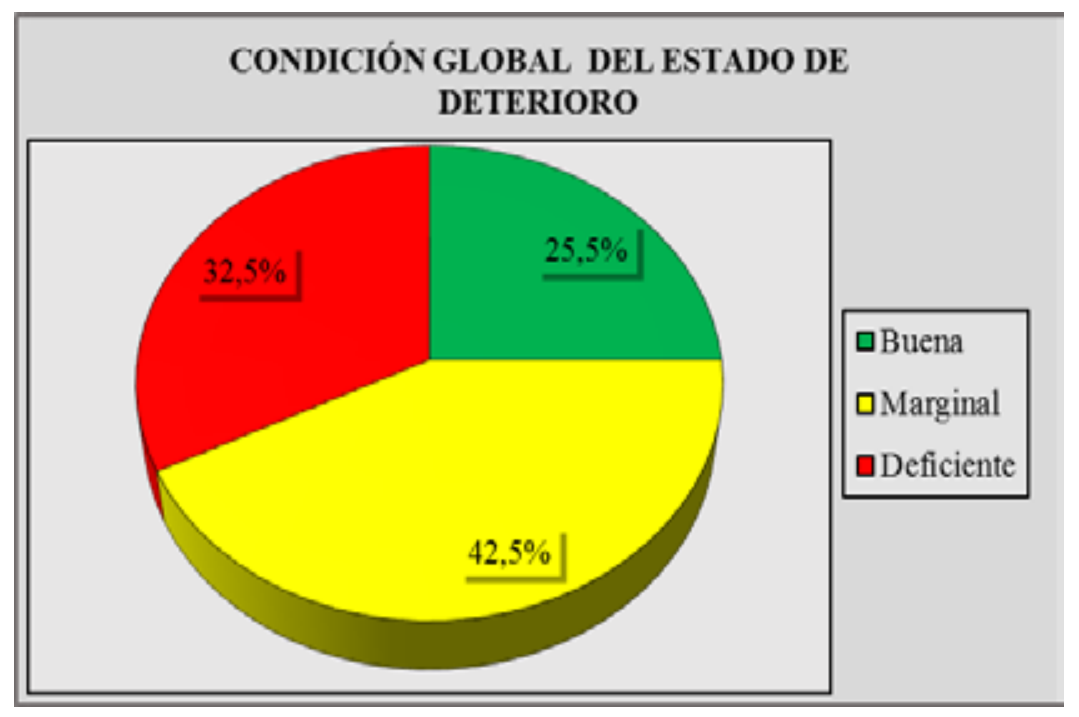

Figura 7. Condición global del estado de deterioro del pavimento asfáltico en el tramo evaluado

Valor de nivel de riesgo por drenaje en el tramo evaluado

La inspección y calificación de la condición del drenaje superficial se obtuvo de acuerdo a los parámetros indicados en el desarrollo. Son los siguientes: Impermeabilidad del revestimiento (R), Drenaje superficial (A) y Ambiente hidrogeológico del pavimento $(\mathrm{H})$. La 
inspección de la condición del drenaje superficial se realizó en los 100 metros de acuerdo a los tramos que evalúa la condición del pavimento de los cuales se obtuvo que la condición de drenaje superficial está en bajo riego según los siguientes resultados de incidencia: Bajo riesgo $60.0 \%$, No hay riesgo $27.0 \%$ y Elevado riesgo $13 \%$.

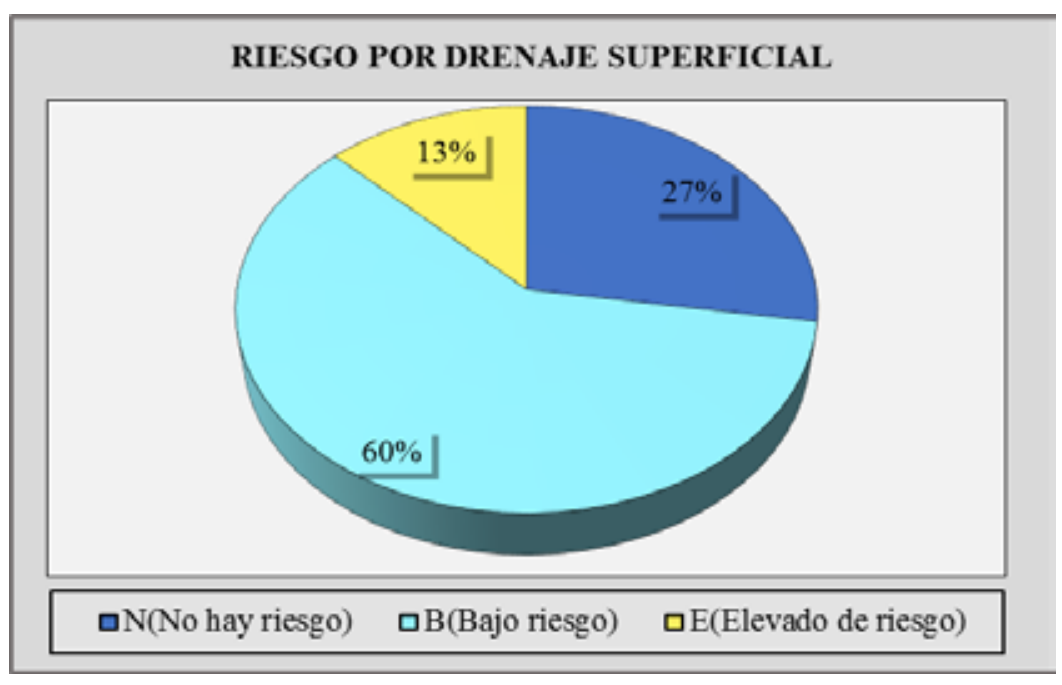

Figura 8. Valores de riesgo por drenaje superficial en la zona de estudio

\section{Alternativas y técnicas de rehabilitación seleccionadas}

El aforo vehicular es también un indicador del tipo de rehabilitación que requiere el pavimento, además de dar mayor certeza de las posibles causas que generan los deterioros. El registro del aforo vehicular se realizó por 12 horas diarias por 7 días para determinar la distribución vehicular en el tramo evaluado. Se consideró 12 horas (7:00 am - 7:00 pm) por el mayor tránsito debido a la restricción del decreto supremo $\mathrm{N}^{\circ}$ 123-2021-PCM Decreto Supremo que declara Estado de Emergencia a Nivel Nacional por las graves consecuencias que afecta a la vida de las personas a consecuencia de la COVID-19, los resultados se presentan en la Tabla 6 .

Tabla 6

Resultados de aforo vehicular por 7 días en el tramo de estudio

\begin{tabular}{ccccc}
\hline \multicolumn{2}{c}{ Resultados Aforo Vehicular } & \multicolumn{2}{c}{ \% según tipo de Vehículo } & Distribución (\%) \\
\hline Tipo de vehículo & IMDs & \% Ligero & \%Pesado \\
Vehículos ligeros & 646 & 71.00 & 71.00 \\
Vehículos pesados & 267 & \multicolumn{2}{c}{ Total (\%) } & 29.00 \\
\hline Transito diario & 913 & \multicolumn{2}{c}{ ) } & 100.00 \\
\hline
\end{tabular}

Del aforo vehicular se ha logrado determinar el volumen de tránsito en ambos sentidos del tráfico, predominando los vehículos ligeros con un $71 \%$ y los vehículos pesados con $29 \%$. Según los resultados obtenidos los futuros deterioros 
estructurales y funcionales en el tramo de estudio (Ramal de Aspuzana - Nuevo Progreso), no solo estarán asociados al tiempo de vida del pavimento o al afecto de las cargas por vehículos pesados, sino que se debe considerar los efectos de riesgo por drenaje superficial.
Las técnicas de rehabilitación fueron seleccionadas de acuerdo a lo considerado en el desarrollo en la Figura 2, además de la selección más acertada de la técnica de rehabilitación también se consideró el tratamiento previo según el tipo de deterioro, como se puede ver en la Tabla 7.

Tabla 7

Técnicas de rehabilitación seleccionadas en el tramo de estudio.

\begin{tabular}{|c|c|c|c|c|}
\hline \multicolumn{2}{|c|}{ Deterioros encontrados } & \multirow{2}{*}{$\begin{array}{l}\text { Símbolo } \\
\text { FLF }\end{array}$} & \multirow{2}{*}{$\begin{array}{c}\begin{array}{c}\text { Tratamiento } \\
\text { previo }\end{array} \\
\text { Geotextiles* }\end{array}$} & \multirow{2}{*}{$\begin{array}{c}\begin{array}{c}\text { Técnica de } \\
\text { rehabilitación }\end{array} \\
\text { Sobrecapa estructural }\end{array}$} \\
\hline $\begin{array}{l}\text { TIPO A } \\
\text { (Condición }\end{array}$ & $\begin{array}{l}\text { Fisuras longitudinales } \\
\text { por fatiga }\end{array}$ & & & \\
\hline Estructural) & Fisuras piel de cocodrilo & FPC & Bacheo & Sobrecapa estructural \\
\hline & $\begin{array}{l}\text { Ahuellamiento y otras } \\
\text { deformaciones estructurales }\end{array}$ & $\begin{array}{l}\text { AH, DL, } \\
\text { DT }\end{array}$ & Bacheo & $\begin{array}{l}\text { Remoción y } \\
\text { reemplazado de capas }\end{array}$ \\
\hline & Bacheos y Parcheos & B & Bacheo & Sobrecapa estructural \\
\hline \multirow{8}{*}{$\begin{array}{l}\text { TIPO B } \\
\text { (Condición } \\
\text { Funcional) }\end{array}$} & $\begin{array}{l}\text { Fisura Long/transv juntas de } \\
\text { construcción }\end{array}$ & FLJ, FTJ & Sello de grietas & $\begin{array}{l}\text { Microaglomerado (en } \\
\text { frío o caliente) }\end{array}$ \\
\hline & $\begin{array}{l}\text { Fisura de contracción } \\
\text { térmica }\end{array}$ & FCT & Sello de grietas & $\begin{array}{l}\text { Microaglomerado (en } \\
\text { frío o caliente) }\end{array}$ \\
\hline & Fisuras de borde & FB & Sello de grietas & $\begin{array}{l}\text { Microaglomerado (en } \\
\text { frío o caliente) }\end{array}$ \\
\hline & Ojos de pescado & $\mathrm{O}$ & Bacheo & Sobrecapa estructural \\
\hline & $\begin{array}{l}\text { Pérdida de la película de } \\
\text { ligante }\end{array}$ & PL & Bacheo & Sobrecapa estructural \\
\hline & Pulimento de agregados & PU & Parcheo & $\begin{array}{l}\text { Microaglomerado (en } \\
\text { frío o caliente) }\end{array}$ \\
\hline & $\begin{array}{l}\text { Desintegración de los bordes } \\
\text { del pavimento }\end{array}$ & DB & Bacheo & Sobrecapa delgada \\
\hline & Erosión de las bermas & EB & Bacheo & Sobrecapa delgada \\
\hline
\end{tabular}

Una vez seleccionada las técnicas de rehabilitación y considerando la Figura 4 de la metodología, se determinó las posibles causas de los deterioros registrados en el tramo de acuerdo a las técnicas de rehabilitación seleccionadas como se puede ver en la Tabla 8 .
Cabe resaltar que las alternativas de rehabilitación seleccionadas pertenecen a los grupos de refuerzo y reconstrucción para tratar adecuadamente las degradaciones del pavimento ocasionados por el tiempo de vida, factores de carga y sobre todo factores climáticos. 


\section{Tabla 8}

Causas de los posibles deterioros registrados en el tramo evaluado

\begin{tabular}{cc}
\hline \multicolumn{2}{c}{ POSIBLES CAUSAS DE DETERIOROS DEL TRAMO } \\
\hline Técnicas de rehabilitación & Causa \\
\hline Microaglomerado (en frío o caliente) & Alta exposición a la humedad \\
\hline Sobrecapa estructural & Alta exposición a la humedad \\
& Drenaje deficiente \\
\hline Remoción y reemplazado de capas & Deficiente compactación base/subbase \\
& Alta exposición a la humedad \\
\hline Sobrecapa delgada & Deficiente compactación base/subbase \\
& Deficiente compactación de subrasante \\
& Drenaje deficiente \\
\hline
\end{tabular}

En el estudio de (Chavez \& Cusquisiban, 2017) se aplicó la metodología VIZIR para plantear técnicas de rehabilitación en el distrito de Ventanilla-Lima, considerando que el pavimento asfáltico evaluado es de categoría regular, siendo vías de bajo tránsito pesado y de precipitación anual mínima se consideró alternativas de rehabilitación por refuerzo y reconstrucción. La principal propuesta está la demolición del pavimento asfáltico en parches de pistas por $5232 \mathrm{~m} 2$.

En otro estudio que realizó (Patarroyo, 2019) sobre evaluación de pavimento asfáltico, determinó el estado de deterioro del pavimento asfáltico como deficiente, además de calificar el riesgo por drenaje en nivel $\mathrm{E}$ (Elevado riesgo). La técnica de rehabilitación que considera es la Reconstrucción de toda la estructura del pavimento.

\section{Mapa de deterioro de pavimentos método VIZIR}

El mapa del estado de deterioro del pavimento en el tramo evaluado contiene los resultados del estado de deterioro del pavimento evaluado con la metodología VIZIR, descritos por colores, el color verde representa al estado bueno, el color amarillo representa al estado marginal y el color rojo representa el estado deficiente. Describe los niveles de riesgo por drenaje superficial, el color azul representa que no hay riesgo, el color celeste representa que el riesgo es bajo y el color amarillo claro representa el elevado riesgo. Además, presenta el clima y la ubicación de las estaciones donde se realizó el conteo vehicular de acuerdo a su código de estación. 


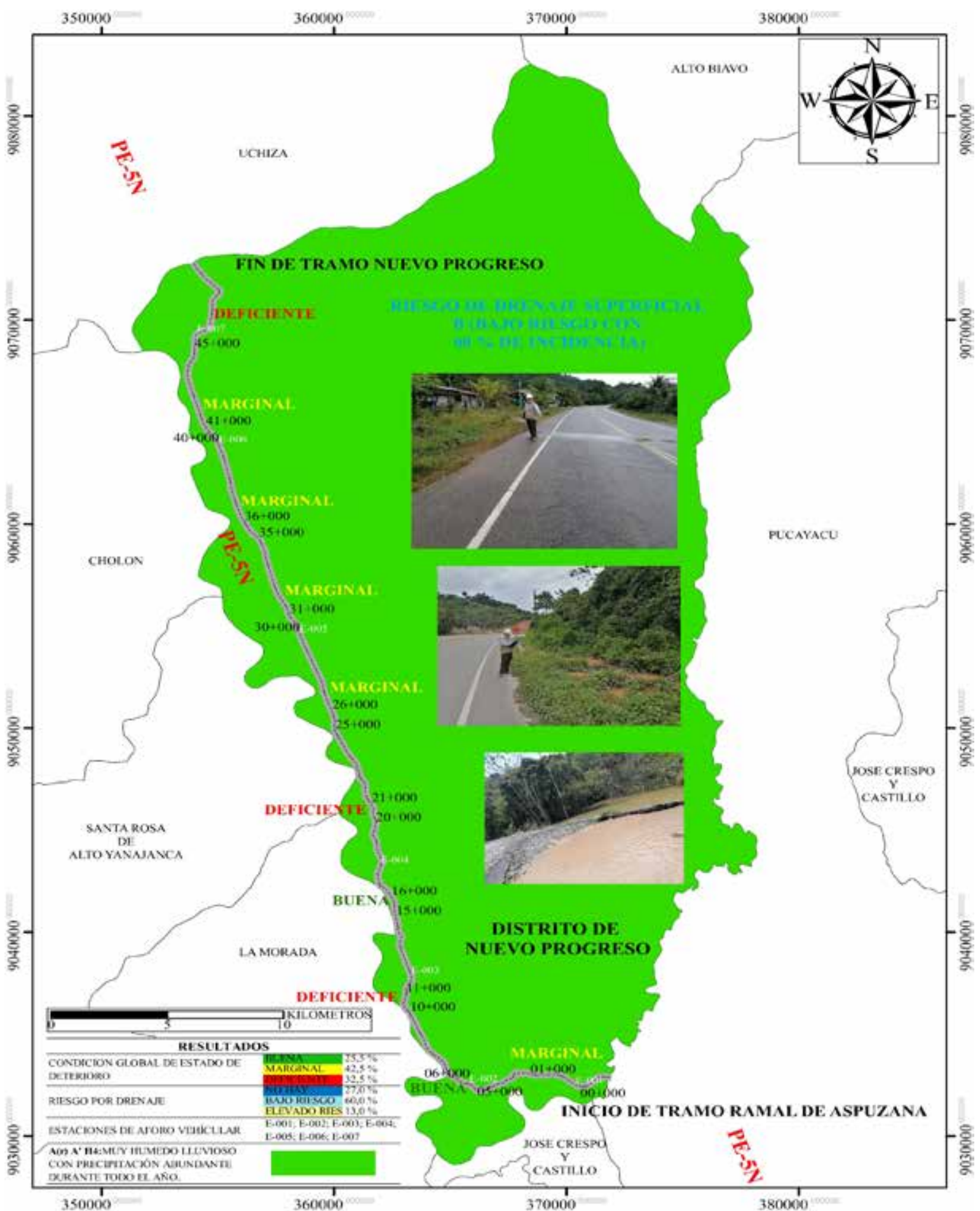

Figura 9. Mapa del estado de conservación

\section{Conclusiones}

El tramo evaluado corresponde desde la localidad de Ramal de Aspuzana a Nuevo Progreso en el distrito de Nuevo Progreso, provincia de Tocache, departamento de San Martín. La inspección de campo se realizó en los meses de abril y mayo de 2021. De acuerdo con la metodología VIZIR, se obtuvo que el índice de deterioro superficial (Is) promedio es 3,68 y la condición global de conservación con mayor incidencia es marginal con $42,5 \%$ siendo el indicador de la condición de 
deterioro del pavimento asfáltico en el tramo evaluado.

El establecimiento de nivel de riesgo por drenaje superficial en el tramo inspeccionado es de Bajo riesgo (B) con un grado de incidencia de $60 \%$, siendo esta una de las principales causas que acelera la gravedad y extensión en los deterioros, sobre todo los deterioros de tipo A, debido al efecto que tiene en la condición estructural del pavimento y los deterioros ojo de pescado del tipo B por su desarrollo acelerado ante la combinación de factores como agua y tránsito pesado.

El volumen de tránsito pesado en el tramo evaluado es solo de $29 \%$ y de tránsito ligero, $71 \%$, por lo que indica que la presencia de los deterioros registrados y los que están en desarrollo no son generados por tránsito pesado ni por el tiempo de vida del pavimento, más bien la condición climática con precipitación promedio anual acumulado de 4489,25 mm está dando señal de su efecto de degradación.

Así mismo, los deterioros que más afectan a la condición del pavimento en el tramo evaluado en función al grado de incidencia son: del tipo A están los Bacheos y Parcheos (B) con $35.6 \%$ esto es debido al mantenimiento rutinario de sello de grietas con asfalto y arena, también están las fisuras de piel de cocodrilo (FPC) con $35.2 \%$ entre sus causas posibles esta la alta exposición a la humedad, drenaje deficiente y errores en el proceso constructivo. Del tipo B, el deterioro que más afecta la condición del pavimento de acuerdo a su grado de incidencia es el pulimento de agregados (PU) con $37.2 \%$. Entre las posibles causas están la alta exposición a la humedad.

\section{Referencias}

Apolinario, E. (2012). Inovación del método VIZIR en estrategias de conservación y mantenimiento de carreteras con bajo volumen de tránsito. Repositorio de Universidad Nacional de Ingeniería.

Chavez, S., \& Cusquisiban, E. (2017). Planteamiento de Estrategias de Rehabilitación del Paviemento Flexible Aplicando la Metodología VIZIR, Para la Optimización de Recursos en la Avenida 225 (Ventanilla-Lima). Repositorio USMP.

Echeverry, C. (2004). Manual de Drenaje de Carreteras. Repositorio de Universidad de los Andes.

Gonzáles, R. (2008). Anexo D: Instructivo para la inspección y la calificación del sistema de drenaje. En Guía metodológica para el diseño de obras de rehabilitación de pavimentos asfálticos de carreteras (págs. 4-8). Instituto Nacional de Vias.

Hidalgo, C. (2006). Evaluación del sistema de gestión de pavimentos flexibles en el Perú. Repositorio de Universidad Peruana De Ciencias Aplicadas.

Instituto Nacional de Vias. (2008). Parte 3: Evaluación de la condición global del paviemnto. En Guía Metodológica para el Diseño de obras de rehabilitación de paviemntos asfálticos de carreteras. (págs. 209211). Ministerio de Transporte. 
Instituto Nacional de Vias. (2008). Parte 4: Selección de técnicas de rehabilitación. En Guía metodológica para el diseño de obras de rehabilitación de pavimentos asfálticos de carreteras (págs. 261263,308,310). Ministerio de Transporte.

INVIAS. (2008). Guía Metodológica para el Diseño de obras de rehabilitación de pavimentos asfálticos de carreteras. Ministerio de Transporte.

Kelly, G., Dalaney, D., Chai, G., \& Mohamed, S. (2014). Optimising local council`s return on investment from annual pavement rehabilitation budgets through targeting of the average pavement condition index. ScienceDirect, 469.

Leguízamo, P. (2008). Anexo B: Instructivo para la inspección visual y la evaluación de los deterioros de los pavimentos asfálticos de carreteras. En Guía metodológica para el diseño de obras de rehabilitación de pavimentos asfálticos de carreteras (págs. 4-36). Instituto Nacional de Vías.

MTC. (2018). Manual de Carreteras Mantenimiento o Conservación Vial. Ministerio de Transportes y Comunicaciones.

Patarroyo, S. (2019). Evaluación de Patologías Método VIZIR en Paviementos Flexibles y Posibles Técnicas de Rehabilitación del Tramo Comprendido entre el KM 8+500 hasta el km 9+000 de la vía Ibagué-Rovira, Departamento de
Tolima. Repositorio Universidad Cooperativa de Colombia.

Porta, S. (2016). Evaluación y comparación de metodologias indice de condición de pavimentos (PCI) y visión $e$ inspección de zonas e itinerarios en riesgo (VIZIR) en la avenida Mariscal castilla tramo: Fundo el Porvenir-La Victoria. Universidad Nacional del Centro del Perú.

Rojas, U. (2011). Evaluación superficial por elmétodo VIZIR carretera CañeteChupaca.Política de mantenimiento. Repositorio de Universidad Nacional de Ingeniería.

SENAMHI. (22 de Junio de 2021). Mapa climatico del Perú. Obtenido de Datos Hidrometeorológicos: https://www.senamhi.gob. pe/?p=mapa-climatico-del-peru

Unidad de Gestión Municipal. (2012). Comparación de métodos de análisis: Notas de calidad (FWD-VIZIR), Notas calidad (FWD-IRI) Y PCI, para escogencia de tipo intervenció en las redes viales cantonales. Universidad de Costa Rica.

VIZIR. (1996). Método con ayuda de computador para la estimación de necesidades en el mantenimiento de una red carretera. LCPC, Laboratoire central des ponts et chaussées.

Zevallos, R. (2018). Identificación y Evaluación de las fallas superficiales en los pavimentos flexibles de algunas vias de la ciudad de Barranca-2017. Repositorio Universidad César Vallejo. 